\title{
Using SPOT Images for Monitoring Water Quality of Reservoir
}

\author{
Po-Hsien $\mathrm{Hsu}^{*}$ \\ Department of Applied Geoinformatics, Chia-Nan University of Pharmacy and Science, \\ No. 60, Sec. 1, Erren Rd., Rende Dist., Tainan City 71710, Taiwan (R.O.C.)
}

(Received August 31, 2015; accepted February 9, 2016)

Keywords: eutrophication, chlorophyll, transparency, total phosphorus, satellite image

Previously, most eutrophic parameters, i.e., chlorophyll, transparency, total phosphorus, of reservoirs were collected manually. Owing to limited budget and time required, the use of eutrophic parameters to reflect water quality was limited. Therefore, in this study, we employed a wide range of multispectral satellite images to carry out water quality monitoring and to understand water quality widely and quickly. To assess eutrophication of water, the most popular standards are the Organization for Economic Cooperation and Development (OECD) and Carlson criteria. In this study, near-infrared, red, and green wavelength spectra of Satellite Pour l'Observation de la Terre (SPOT) images were applied to monitor three parameters. Using ERDAS Imagine software (designed by ERDAS, Inc. for geospatial applications) for data analysis and then using the software of Statistical Product and Service Solutions (SPSS) to seek more appropriate regression formulas, the satellite image data were converted into water quality parameters on-site and used to judge the water quality by the criteria of OECD and Carlson indicators. By comparison, the results between collecting from satellite images and on-site by hand were quite similar except for total phosphorus, which might result from unsuitable wavelength spectra from SPOT. Further studies are necessary.

\section{Introduction}

In this study, the Satellite Pour l'Observation de la Terre (SPOT) images, which included three bands - near-infrared, red, and green light-were applied to detect water quality, i.e., chlorophyll density, Secchi disk depth, and total phosphorus density. The ground resolution of the SPOT multifrequency images is $20 \times 20 \mathrm{~m}^{2}$; that of the pan-chromatic images is $10 \times 10 \mathrm{~m}^{2}$. The relationship among the density of Secchi's disk depth, chlorophyll, phosphorus, and other substances to the spectral reflectance value required investigation, since the numerical results of images defined the strength of spectral reflectance.

By comparison with the collected data from on-site investigation as the traditional approach in the water area sampled, regression formulas could be established. Using these formulas, the spectral reflectance data over a wide range of reservoirs could be estimated. Then the relationship between the values and the water parameters collected on-site could be evaluated. Then, using the evaluation method to analyze the eutrophication index throughout the reservoir and to determine the extent of the reservoir eutrophication, it was possible to establish a more suitable model for

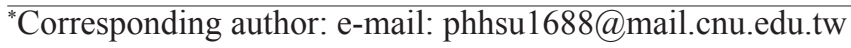


water quality evaluation of the eutrophication index and classification in Taiwan to provide a reference for water quality management. Using satellite imagery and analysis that have a variety of ways to monitor water quality was an effective way to address the shortcomings of traditional water quality monitoring. If the relationship between spectral characteristics of water reflection and density of water quality could be realized, and the calculation of water quality parameters could be established, then one could obtain the advantages of wide-range monitoring and higher efficiency of data collection.

\section{Materials and Methods}

In this study, SPOT-4 satellite images were employed for analyzing water quality and establishing a regression model (shown in Fig. 1). There are three multispectral bands, i.e., green light (G1: $0.5-0.59 \mathrm{~mm})$, red light $(R 2: 0.61-0.68 \mathrm{~mm})$, and near-infrared light (NI3: 0.79-0.89 $\mathrm{mm}$ ), each with a spectral resolution of $20 \mathrm{~m}$. The collected sample data, i.e., transparency, chlorophyll, and total phosphorus, on-site at five stations, i.e., Crest, Xiandao, Pearl Bay, Armuping, and Changxing, around the Shihmen Reservoir corresponded to the same day the satellite images were taken and were used to form a regression model of this area.

From the relationship between the spectral reflection data and the data collected on-site, a model of regression formulas could estimate data over a wide range in the reservoir to evaluate the degree of eutrophication. The most popular criteria for evaluating water quality are the Organization for Economic Cooperation and Development (OECD) environmental indicators and the Carlson index. ${ }^{(1)}$ They were the evaluation criteria used for this study. The procedures for this study were as follows.

\subsection{Image correction, registration, and calculating band reflectance}

The band reflectance value of each station was calculated from the average of the numerical value of the sample at the central grid and from eight adjacent regular grids which were $20 \times 20 \mathrm{~m}^{2}$ in size. The average value of three bands, i.e., green light $(G 1)$, red light $(R 2)$, and near-infrared light (NI3), was calculated. Since the images were not accurately located in terms of their positions when they were superimposed, it was necessary for further pinpointing to ensure that their positions were not far from the sampling station.

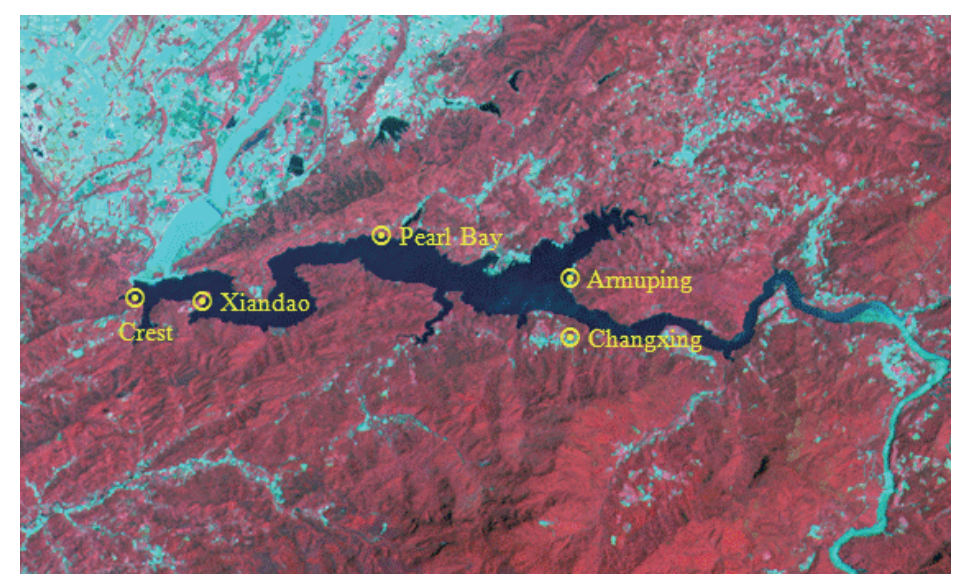

Fig. 1. (Color online) Satellite image of Shihmen Reservoir. 


\subsection{Establishing the regression model}

The regression formulas were derived for the conversion of imaging data for comparison to the data collected on-site, which aimed to establish values at the site for the three parameters, i.e., chlorophyll, total phosphorus, and transparency. The formulas were highly relevant to spectral reflectance for improving the reliability of satellite image monitoring of water quality. The natural logarithm for conversion was derived from the regression equation and was expressed as follows: ${ }^{(2)}$

$$
\ln Y_{i}=a_{i}+a_{i j} \ln B_{j}
$$

where $Y_{i}$ is the on-site value of water quality parameters $i, B_{j}$ represents the ratio of the two spectral bands, and $a_{i}$ and $a_{i j}$ are constant values determined by linear regression.

Satellite images of the Shihmen Reservoir received in August 2008 and the manually collected data on-site from the same day were used to develop a regression formula. In the application of water quality eutrophication assessment, chlorophyll, Secchi disk depth, and total phosphorus were employed to determine the degree of eutrophication. The ratio of the red and near-infrared bands has a high correlation with chlorophyll. ${ }^{(3)}$ Also, according to Lathrop and Lillesand in 1989, the Secchi disk depth had a high correlation to the ratio of green light to red light, and in terms of the total phosphorus concentrations, the ratio of green light to infrared light had a high degree of correlation. ${ }^{(4)}$

The first step employed the average data from selected images and the data collected on-site to generate a regression model to calculate the chlorophyll (CHLA), total phosphorus (TP), and transparency $(S D)$. The second step searched the most appropriate regression formula under the criteria that considered $R^{2}$ values as close as possible to 1 and $P$ values less than 0.05 to verify if the formula was acceptable. Finally, a regression equation was selected to convert existing water quality parameters. The selected formulas for OECD are as follows:

$$
\ln (C H L A)=22.385-11.167 \times(N I 3 / R 2)-1.873 \times(G 1 / N I 3)-15.956 \times(R 2 / G 1)
$$

where $R^{2}=0.995$ and $P=0.090$,

$$
\ln (S D)=-7.158+1.409 \times(G 1 / R 2)+1.464 \times \ln (R 2)
$$

where $R^{2}=0.852$ and $P=0.148$, and

$$
\ln (T P)=68.840-16.759 \times \ln (G 1)
$$

where $R^{2}=0.786$ and $P=0.045$.

Then using the Carlson multivariate formula, chlorophyll, total phosphorus, and transparency, are shown as follows:

$$
\begin{gathered}
C H L A=9.81 \times \ln (C H L A)+30.6, \\
S D=60-14.41 \times \ln (S D), \\
T P=14.42 \times \ln (T P)+4.15 .
\end{gathered}
$$


The Carlson trophic state index (CTSI) was based on the values obtained in the multivariate index, which is shown as follows:

$$
C T S I=[\operatorname{TSI}(C H L A)+\operatorname{TSI}(S D)+\operatorname{TSI}(T P)] / 3 .
$$

\section{Results}

The satellite image was separated into three layers, i.e., green, red, and near-infrared light, using ERDAS software and the imaging data was converted into water quality parameters by appropriate regression formulas derived using the software of Statistical Product and Service Solutions (SPSS). ${ }^{(5,6)}$ After transforming to water quality parameters, the output files of images were judged by OECD eutrophication criteria (see Table 1) and the CTSI (see Table 2) to give different degrees of eutrophication graded colors as shown in Figs. 2 and 3, respectively. From Fig.

Table 1

OECD eutrophication criteria (Yang et al. 2008).

\begin{tabular}{lccc}
\hline Grade & $\mathrm{TP}(\mu \mathrm{g} / \mathrm{l})$ & CHLA $(\mu \mathrm{g} / \mathrm{l})$ & $\mathrm{SD}(\mathrm{m})$ \\
\hline Oligotrophic $(\mathrm{O})$ & $<7.9$ & $<2$ & $>4.6$ \\
Oligotrophic-Mesotrophic (O-M) & $8-11$ & $2.1-2.9$ & $3.8-4.5$ \\
Mesotrophic (M) & $12-27$ & $3-6.9$ & $2.4-3.7$ \\
Mesotrophic-Eutrophic (M-E) & $28-39$ & $7-9.9$ & $1.8-2.3$ \\
Eutrophic (E) & $>40$ & $>10$ & $<1.7$ \\
\hline
\end{tabular}

Table 2

CTSI (Yang et al. 2008).

\begin{tabular}{lccc}
\hline Grade & $\mathrm{TP}(\mu \mathrm{g} / \mathrm{l})$ & $\mathrm{CHLA}(\mu \mathrm{g} / \mathrm{l})$ & $\mathrm{SD}(\mathrm{m})$ \\
\hline Oligotrophic $(\mathrm{O})$ & $<12$ & $<2.6$ & $>4$ \\
Mesotrophic $(\mathrm{M})$ & $12-24$ & $2.6-7.2$ & $2-4$ \\
Eutrophic (E) & $>24$ & $>7.2$ & $<2$ \\
\hline
\end{tabular}

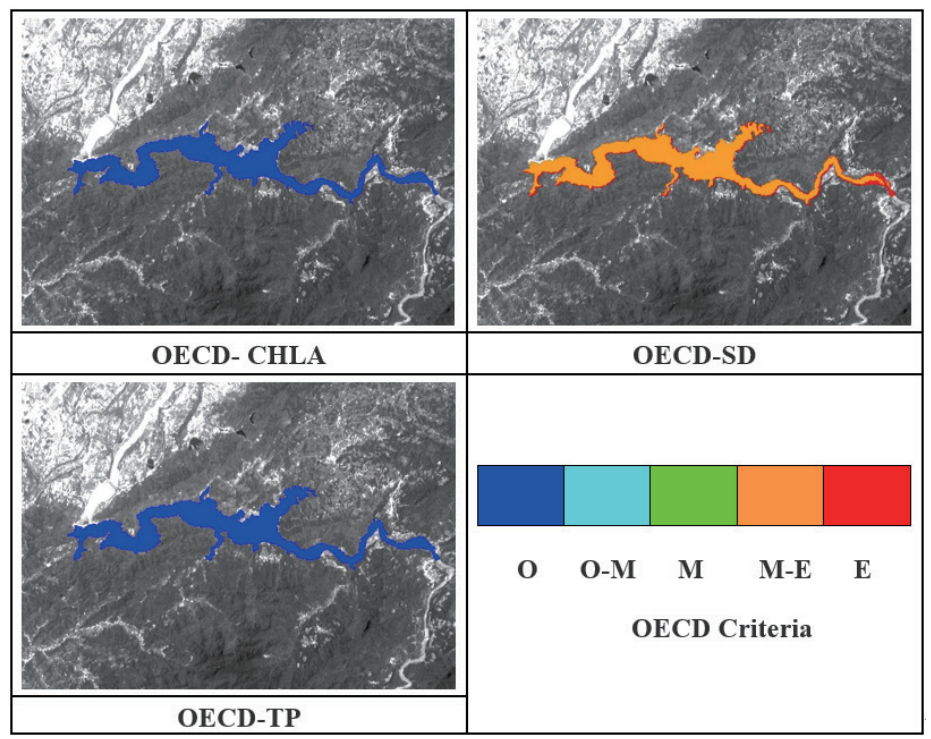

Fig. 2. (Color online) Satellite image of OECD in August 2008. 


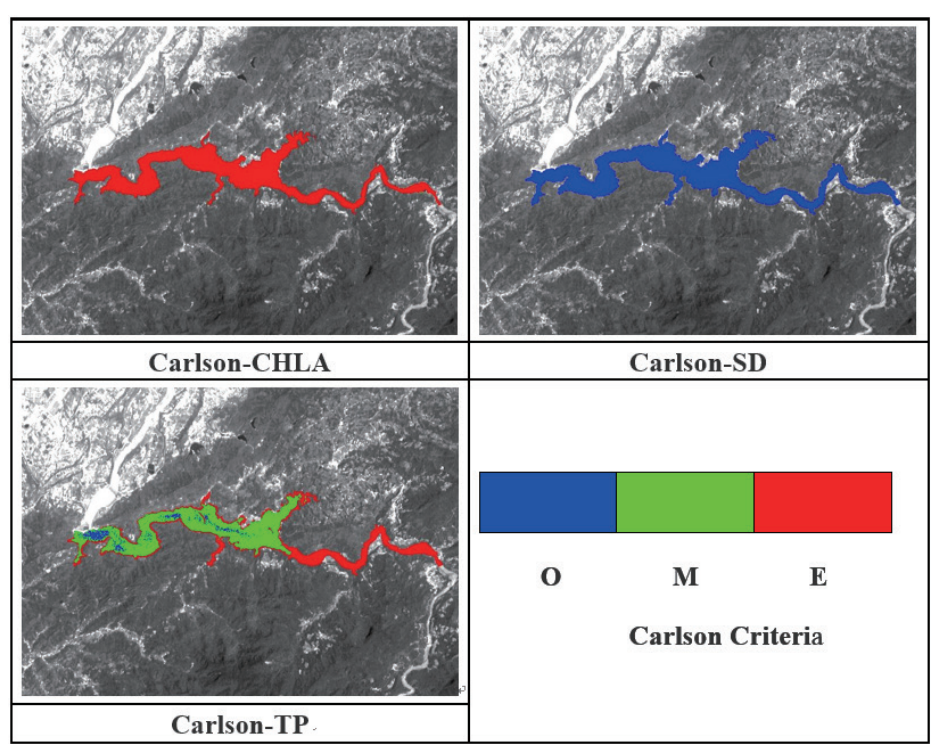

Fig. 3. (Color online) Satellite image of TSI in August 2008.

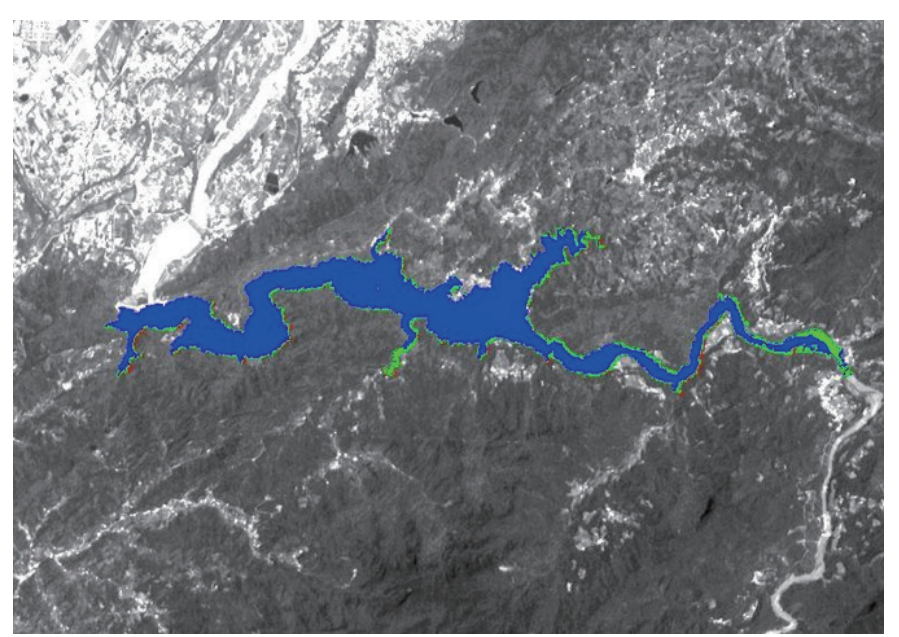

Fig. 4. (Color online) Satellite image of CTSI in August 2008.

2, the water quality judged by the OECD was between oligotrophic and mesotrophic. From Fig. 3 , the water quality judged by the Carlson index was between oligotrophic and eutrophic. Finally, the CTSI was used to assess water quality. If $C T S I<40$, the water is in an extremely oligotrophic condition, and if CTSI $>50$, it is in an extreme state of eutrophication. Between the two states, the water quality is in an ordinary state. The water quality judged by CTSI was between oligotrophic and mesotrophic (shown in Fig. 4). After calculating the inverse value of the image, the average of five sampled points, and the average values for the month, the average values for the month were then compared with the sampled value on-site as shown in Figs. 5-7. 


\section{CHLA of OECD}

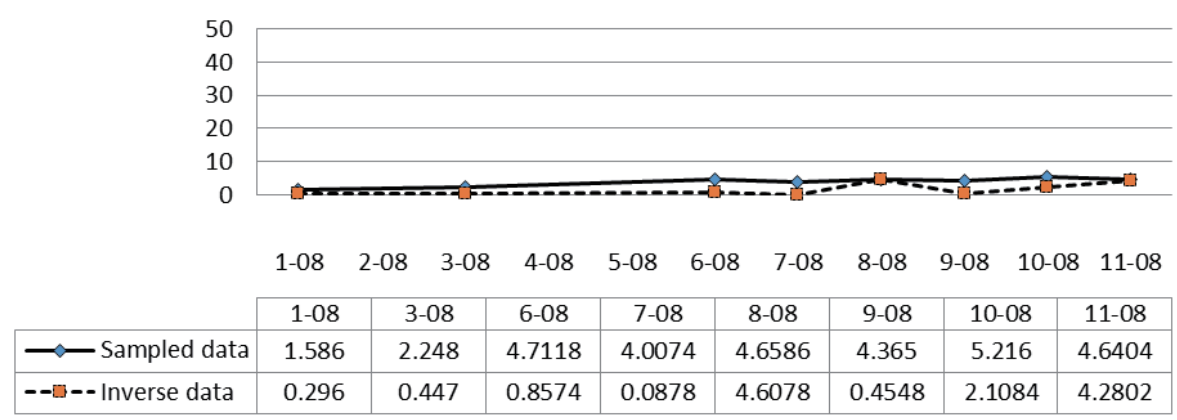

Fig. 5. (Color online) Differences in CHLA between the average values for the month and the sampled data onsite.

\section{SD of OECD}

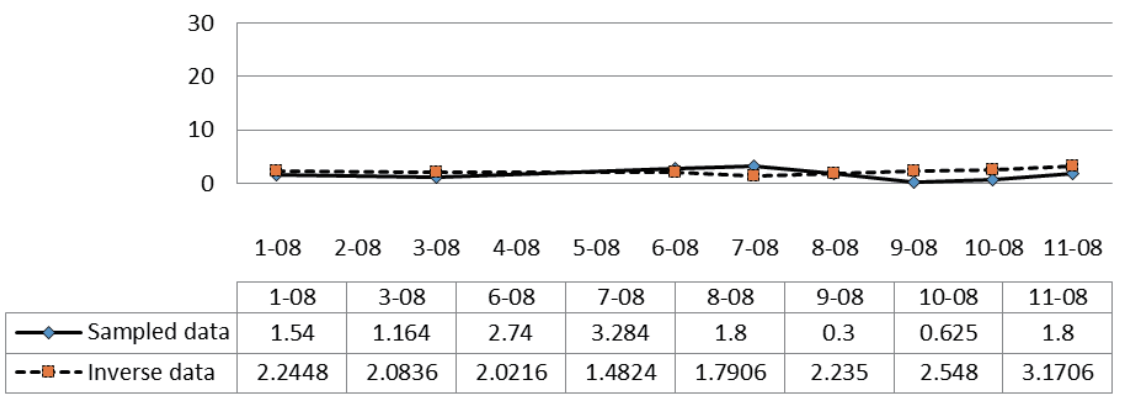

Fig. 6. (Color online) Differences in SD between the average values for the month and the sampled data on-site.

\section{TP of OECD}

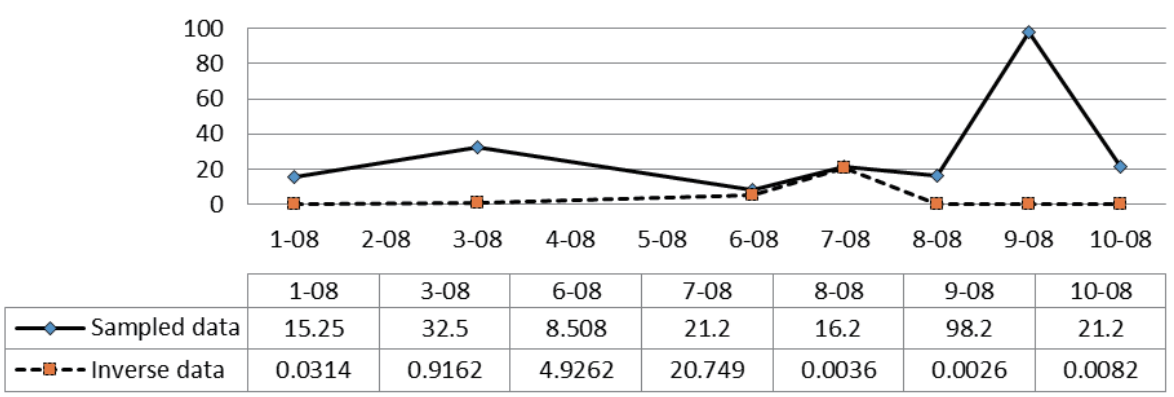

Fig. 7. (Color online) Differences in TP between the average values for the month and the sampled data on-site. 


\section{Discussion}

Figure 2 shows the colors of water quality parameters, i.e., CHLA, SD, and TP in OECD criteria. The colors of CHLA and TP are in blue or oligotrophic. The color of SD is brown or mesotrophic-eutrophic due to a rainstorm which generated a mixing between upper and lower water layers. Based on the OECD criteria, the water quality can be qualified as in good condition. Figure 3 shows the colors of water quality parameters in the Carlson criteria. Except for SD, the water quality in terms of CHLA and TP is poor. Usually, instead of Carlson criteria, the multivariate index of the CTSI is used to assess water quality. Figure 4 is based on the CTSI and shows the water quality is still good. Figures 5-7 show the differences between the average values of five sampled points and the sampled values on-site for the month. The differences in the distribution of CHLA and SD shown in Figs. 5 and 6 are quite consistent, but the differences in the distribution of TP in Fig. 7 are not. Data processing of SPOT images may not be suitable for determining TP.

\section{Conclusions}

From this study, it is confirmed that using satellite images is a quick and effective method to monitor a large area of water quality for eutrophication. The results based on eutrophication parameters, i.e., chlorophyll and transparency, defined using satellite images is quite consistent with the values determined on-site.

Because of the eutrophication of water at high temperature or during the change of seasons, the water generates upper and lower water layers. If only one month of data is used, it is difficult to determine whether the water quality is good or not. Therefore, it is necessary to analyze a full year of satellite imaging data to evaluate water quality.

When using SPSS to find appropriate regression formulas, the requirements are for $R^{2}$ to be as close to 1 as possible and for $P$ values to be less than 0.05 . Sometimes the data may be consistent with the on-site value when a satellite image is converted to an on-site value, but it often cannot be converted into suitable values, because the spectral data are more complex. Therefore, when looking for regression formulas, one must test very carefully to find the best formulas.

\section{Acknowledgements}

The author thanks to the administration of Shihmen Reservoir for providing the on-site data for chlorophyll, transparency, and total phosphorus in the reservoir and Professor Yang of the University of Chung-Hsing for providing the SPOT images.

\section{References}

1 M. D. Yang, Y. C. Liu, and K. S Huang: The 17th Conference of Hydraulic Engineering (Taiwan, 2008) pp. M2-1-M2-7.

2 R. G. Lathrop and T. M. Lillesand: Photogrammetric Eng. Remote Sens. 52 (1986) 671.

3 M. D. Yang, C. J. Merry, and R. M. Sykes: J. Am. Water Resour. Assoc. 35 (1999) 253.

4 R. G. Lathrop and T. M. Lillesand: Photogrammetric Eng. Remote Sens. 55 (1989) 349.

5 M. M. Avard, F. R. Schiebe, and J. H. Everitt: Photogrammetric Eng. Remote Sens. 66 (2000) 213.

6 T. M. Lillesand, W. L. Johnson, R. L Deuell, O. M. Lindstorm, and D. E. Meisner: Photogrammetric Eng. Remote Sens. 49 (1983) 219. 


\section{About the Author}

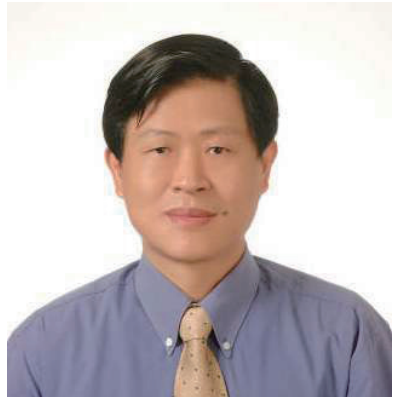

Po-Hsien Hsu received his B.S. degree from Chung-Cheng Institute of Technology, Taiwan ROC, in 1977, his M.S.E. degree from University of Washington, USA, in 1986, and his Ph.D. degree from University of Nottingham, UK, in 1996. He was an assistant professor at Chinese Military Academy, Taiwan. Since 2007, he has been an associate professor at Chia-Nan University. His research interests are in 3S. 